\title{
Experimental Study on the Segmental Innervation of the Spinal Cord to the Visceral Organs
}

\author{
Sentaro Shishito \\ Sendai Social Insurance Hospital, Sendai 980
}

\begin{abstract}
Sнrsнтт, S. Experimental Study on the Segmental Innervation of the Spinal Cord to the Visceral Organs. Tohoku J. exp. Med., 1978, 125 (1), 19-30To clarify the segmental innervation of the spinal cord to the visceral organs, selective section of the individual spinal nerve root of the dog in different spinal segment was performed, and the distribution of distal degeneration of myelinated nerve fibers in the sympathetic trunk and in peripheral nerves was observed. From the results obtained, much wide-spread innervation of each spinal segment to the visceral organs was revealed, and an efferent system in the dorsal root of the spinal nerve was confirmed to be related to the visceral organ. Furthermore, widely extended innervation of the vagus nerve to the visceral organ was clarified. - spinal innervation to visceral organ; degeneration of myelinated nerve fiber; autonomic innervation; vagal innervation; spinal parasympathetics
\end{abstract}

It is generally accepted that visceral organs receive their proper innervation from the spinal nerves, directly or indirectly via the sympathetic trunk, and partly from the vagus nerve. However, the mode of segmental innervation of the spinal cord to these organs is rather obscure, so that it sometimes causes confusion with regard to the centripetal pathway of the visceral pain from these organs as well as the autonomic influence to these tissues. To clarify such a problem, therefore, selective section of individual spinal nerve root of the dog in different spinal segment was performed, and peripheral distribution of the distal degeneration of myelinated nerve fibers was pursued histologically.

\section{Matertals and Methods}

Mongrel dogs of both sexes, weighing $7-10 \mathrm{~kg}$, were used in this experiment. Selective section of the spinal nerve was performed carefully according to the routine procedure on the ventral root, dorsal root, and spinal ganglion of the thoracic, lumbar, and sacral nerves, expecting an appearance of distal degeneration of myelinated nerve fibers in peripheral region.

One week after the nerve section, available specimens of peripheral nerve bundles were extirpated from the nerves just on visceral organs as well as from the sympathetic trunk, and fixed in $0.2 \%$ osmic acid solution for $24 \mathrm{hr}$ at $30^{\circ} \mathrm{C}$. Then it was splitted into every single nerve fiber with microscopic manipulation described by Fukuyama (1955), and the number of the myelinated nerve fibers, degenerated or non-degenerated, was counted.

Received for publication, August 8, 1977.

This paper is presented in commemoration of the 60th anniversary of the First Department of Surgery, Tohoku University School of Medicine. 


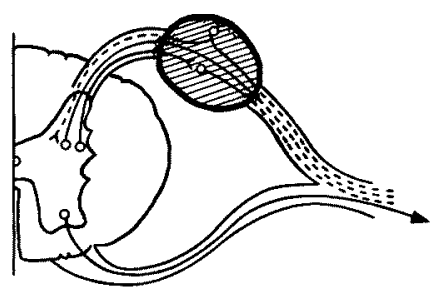

Spinal gonglionectomy
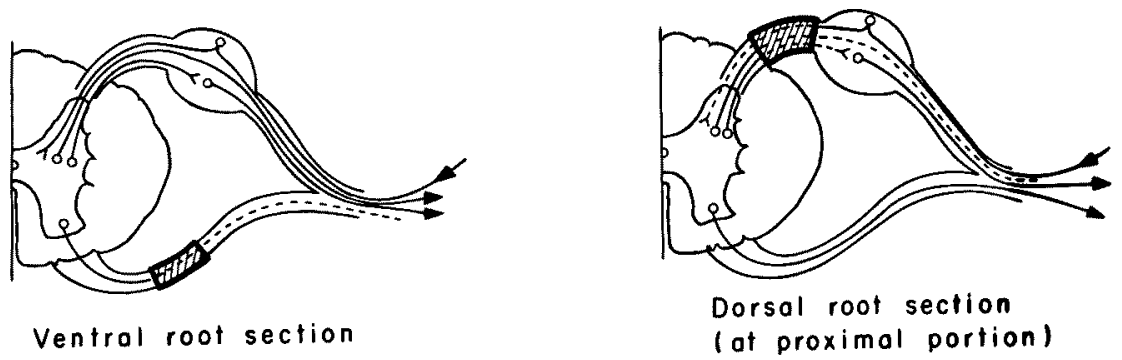

Fig. 1. Degeneration of the myelinated nerve fibers after the section of the spinal nerve.

The centrifugal degeneration of the myelinated nerve fiber after selective section of the spinal nerve root was assumed to be as shown in Fig. 1. And the spinal origin of the nerve fiber was suggested from the relation between the mode of nerve section and nerve degeneration. Furthermore, the peripheral distribution of degenerated nerve fibers after the section of the dorsal root at the proximal part from the spinal ganglion might reveal the efferent pathway of spinal parasympathetic system through the dorsal root of the spinal nerve, as suggested by Kure and Okinaka (1949) and reported by Shishito and Suzuki (1961).

\section{Results AND Discussion}

The distribution modes of degenerated nerve fibers in various parts of the sympathetic trunk one week after selective section of the spinal nerve root, which were reported by Chan (1957), Irisawa (1959), Maeda (1959), Yambe (1960), Inomata (1960), Funada (1960), Kano (1958), and Kashimura (1959), and supported by the results reported by Kikuchi (1955), Haruyama (1958), Kato (1960), Sakauchi (1960) and Imabayashi (1971), are summarized in Fig. 2 (spinal ganglionectomy), Fig. 3 (ventral root section), and Fig. 4 (dorsal root section). From these results, it was clarified that one spinal segment has rather wide-spread contact with several sympathetic ganglia, not only in afferent but also in efferent pathway including the efferent system in the dorsal root.

On the other hand, the peripheral distribution of degenerated nerve fibers to various visceral organs was observed in the same way. The results obtained from the lung (Iizuka 1959), stomach (Hara 1957; Muto 1960; Ono 1960), liver and gall 


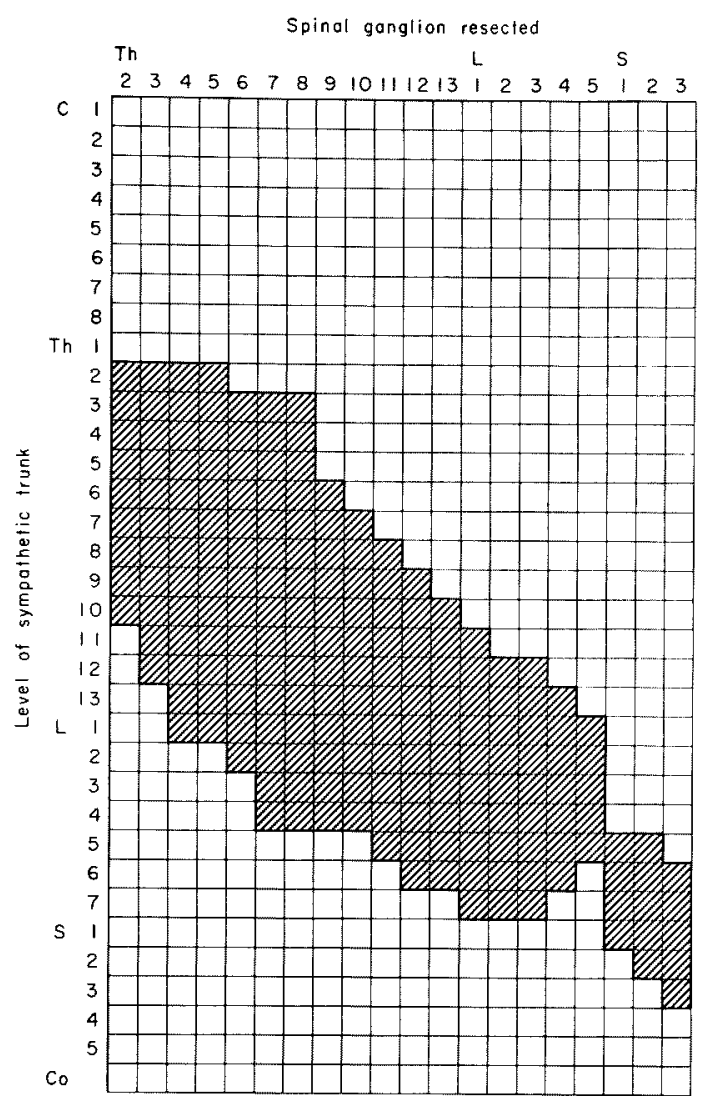

Fig. 2. The distribution of the myelinated nerve fibers degenerated after the spinal ganglionectomy in the sympathetic trunk. The hatched area shows the presence of degenerated fibers.

bladder (Honda 1960; Inomata 1957), pancreas (Koseki 1958), spleen (Watanabe 1959), small intestine (Suzuki 1958), colon (Yoshinari 1959), rectum (Sakata 1961), adrenal glands (Mashiko 1959), kidney (Tokoyo 1959), ureter (Okane 1960), urinary bladder (Sato 1957; Saito 1958; Kimura 1960), male internal sexual organs (Okuno 1958), and female internal sexual organs (Okuno et al. 1958), are summarized in Fig. 5 (spinal ganglionectomy), Fig. 6 (ventral root section), and Fig. 7 (dorsal root section).

Figs. 2-7 show a distribution range of myelinated nerve fibers in the sympathetic trunk or in peripheral nerves, degenerated after causal nerve section. At the same time, these results would reveal the relation of those degenerated nerve fibers to the original spinal segment. However, these results include all of the small-, medium-, large-, and maximum-sized myelinated nerve fibers. As to the size and function of nerve fibers in the splanchnic nerve, Osaki (1956) assumed that the small-sized myelinated fibers, which have rather thin myelin sheath, short distance in each node of Ranvier, and slight Schmidt-Lantermann incisure, might 


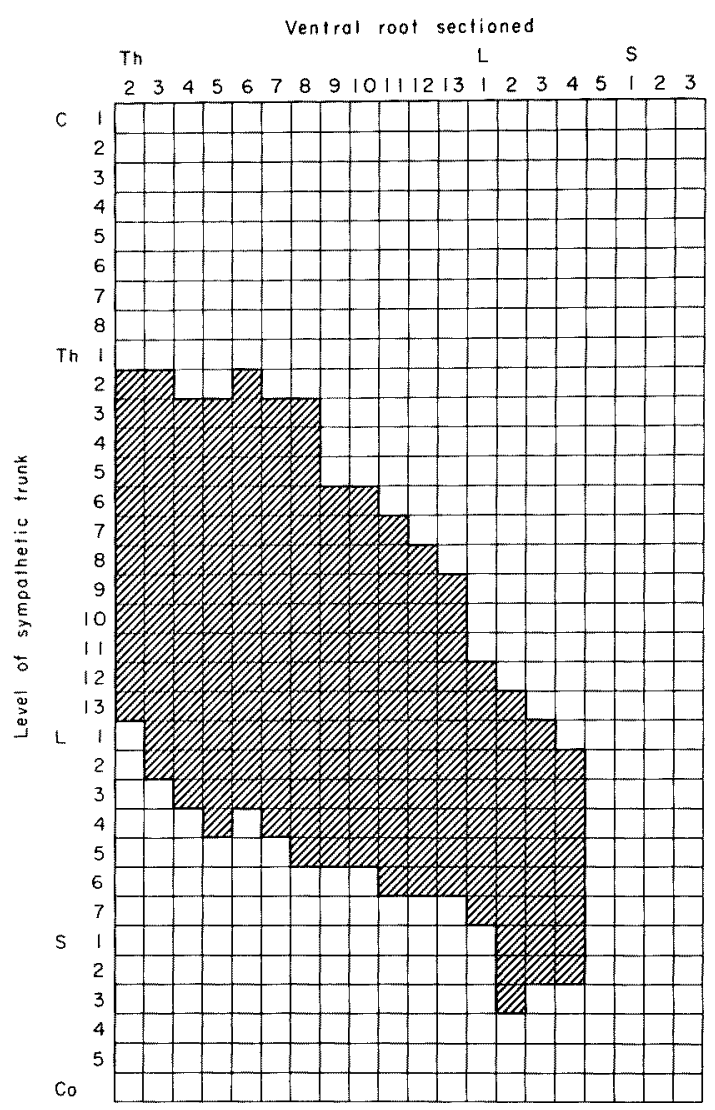

Fig. 3. The distribution of the myelinated nerve fibers after the section of the ventral root in the sympathetic trunk. The hatched area shows the presence of degenerated fibers.

be autonomic in its nature. And Hamabe (1932) had discussed upon the relationship between the diameter of the myelinated nerve fibers and the pathway of sensation. Furthermore, Sato et al. (1957) had stressed a significant meaning of the diameter of the myelinated nerve fivers relating to the visceral sensation. Therefore, we examined the small-sized fibers in more detail. The results obtained are shown in Fig. 8 (spinal ganglionectomy), Fig. 9 (ventral root section), and Fig. 10 (dorsal root section). We can see clearly the appearance of the small-sized nerve fibers degenerated in the peripheral portion of its pathway. The results shown in Fig. 9 might reveal the autonomic efferent system, but the results shown in Fig. 8 might include both the afferent and the efferent system of the dorsal root, and the results shown in Fig. 10 might reveal the site of the efferent system in the dorsal root of the spinal nerve.

The real number of degenerated nerve fibers was quite different from each other in Figs. 2-4, Figs. 5-7, and Fig. 8-10, as well as in every segment of the 


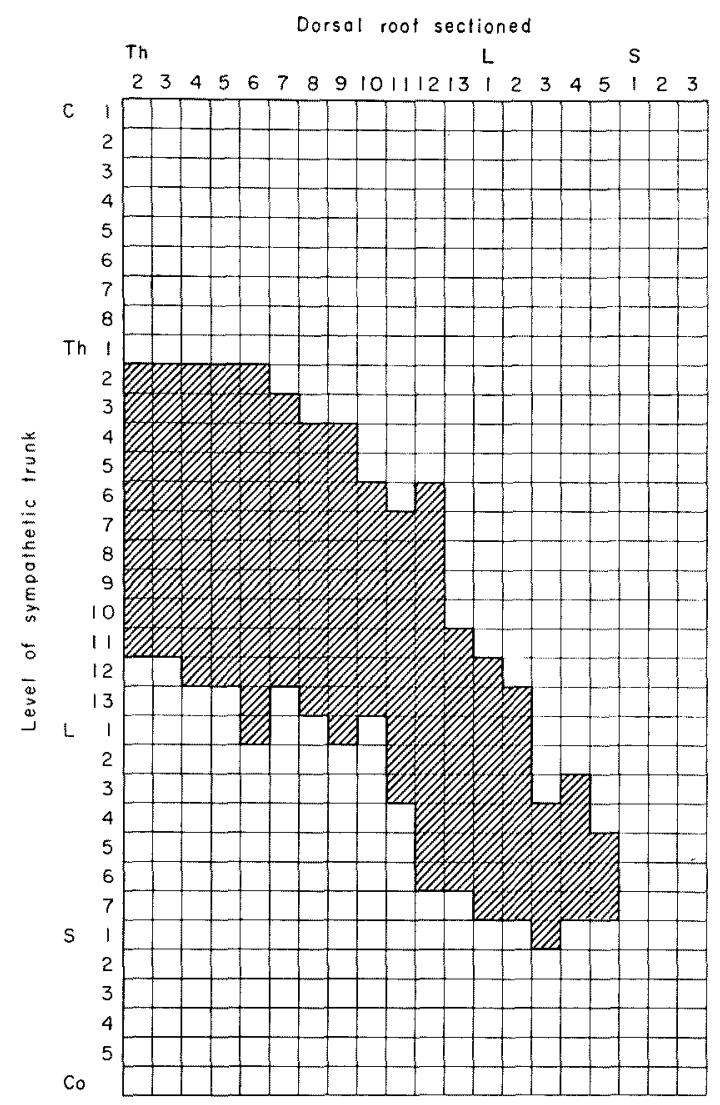

Fig. 4. The distribution of the myelinated nerve fibers after the section of the dorsal root in the sympathetic trunk. The hatched area shows the presence of degenerated fibers.

TABLE 1. The distribution of the degenerated nerve fivers to the visceral organ after the section of the vagal system

$$
\text { A } B
$$

\begin{tabular}{|c|c|c|c|c|c|c|c|c|c|c|}
\hline \multirow{3}{*}{ Organ } & \multirow{2}{*}{\multicolumn{5}{|c|}{ Size of nerve fiber }} & \multirow{2}{*}{\multicolumn{5}{|c|}{ Size of nerve fiber }} \\
\hline & & & & & & & & & & \\
\hline & Small & Medium & Large & Maximum & Total & Small & Medium & Large & Maximum & Total \\
\hline Stomach & 109 & 19 & 0 & 0 & 128 & 219 & 66 & 0 & 0 & 285 \\
\hline Liver & 3 & 1 & 0 & 0 & 4 & 21 & 5 & 0 & 0 & 26 \\
\hline $\begin{array}{l}\text { Gall } \\
\text { bladder }\end{array}$ & 1 & 1 & 0 & 0 & 2 & 2 & 0 & 0 & 0 & 2 \\
\hline Spleen & 1 & 0 & 0 & 0 & 1 & 2 & 1 & 0 & 0 & 3 \\
\hline $\begin{array}{l}\text { Small } \\
\text { intestine }\end{array}$ & 11 & 4 & 0 & 0 & 15 & 15 & $\overline{2}$ & 0 & 0 & 17 \\
\hline $\begin{array}{l}\text { Mesenteric } \\
\text { plexus }\end{array}$ & 0 & 0 & 0 & 0 & 0 & 0 & 0 & 0 & 0 & 0 \\
\hline $\begin{array}{l}\text { Large } \\
\text { intestine }\end{array}$ & 1 & 1 & 0 & 0 & 2 & 2 & 1 & 0 & 0 & 3 \\
\hline Kidney & 3 & 1 & 0 & 0 & 4 & 2 & 1 & 0 & 0 & 3 \\
\hline Adrenals & 5 & 1 & 0 & 0 & 6 & 0 & 9 & 0 & 0 & 9 \\
\hline Pancreas & 10 & 1 & 0 & 0 & 11 & 2 & 0 & 0 & 0 & 2 \\
\hline
\end{tabular}

A: Section of the ventral and the dorsal stem of the vagus just below the diaphragm.

B: Bilateral section of the vagus just below the nodose ganglion. 


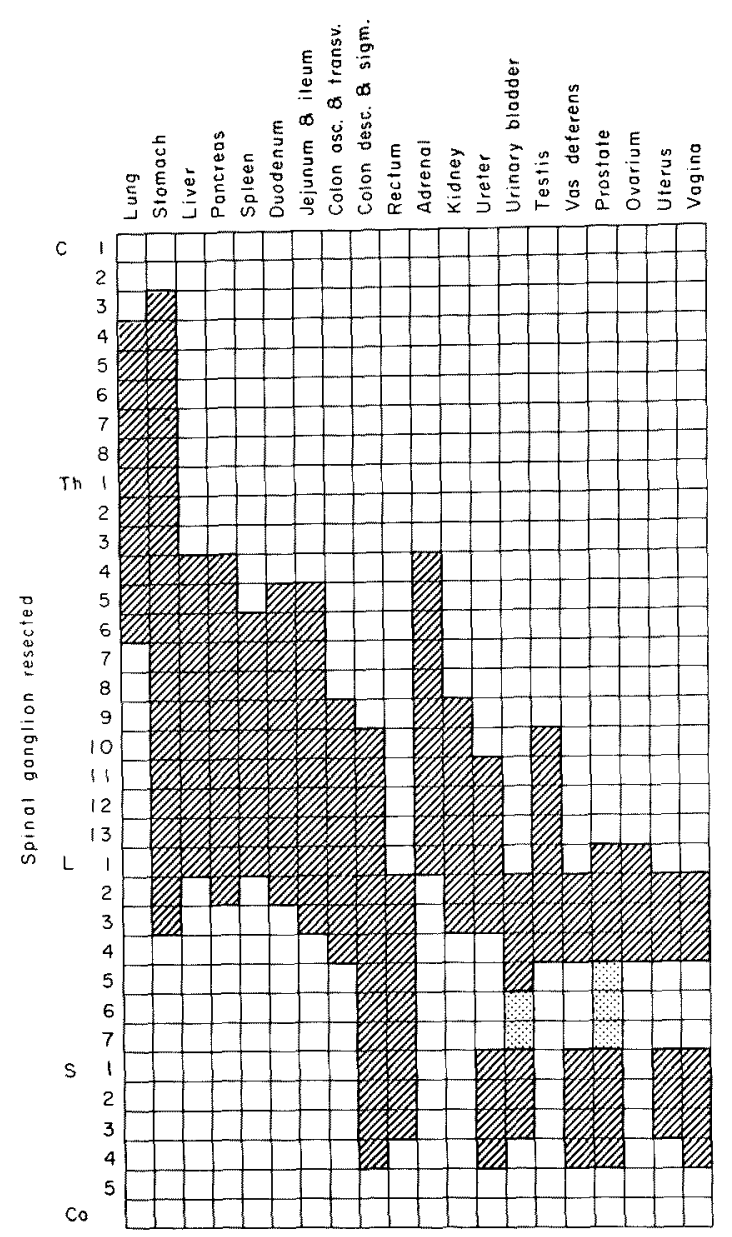

Fig. 5. The distribution of the degenerated nerve fibers to the visceral organ after the spinal ganglionectomy. The hatched area shows the presence of dgenerated fibers.

sympathetic trunk and every peripheral nerve. As suggested by Sato (1957), it should be thought that most of the preganglionic efferent nerve fibers from the spinal nerve root might change to the postganglionic fibers which have no myelin sheath in general. A marked difference in number was found between the degenerated nerve fibers in the sympathetic trunk and those in peripheral nerves, but it was thought that the appearance of degenerated nerve fibers in the peripheral nerve might reveal the direct relation between the sectioned nerve root and the peripheral nerve innervating the visceral organ, even if the number of the degenerated fibers was rather small in the latter.

On the other hand, the vagal innervation to the visceral organ was examined. The nerve section was performed on the ventral and the dorsal stem of the vagus just below the diaphragm, and also the bilateral vagus nerve just below 


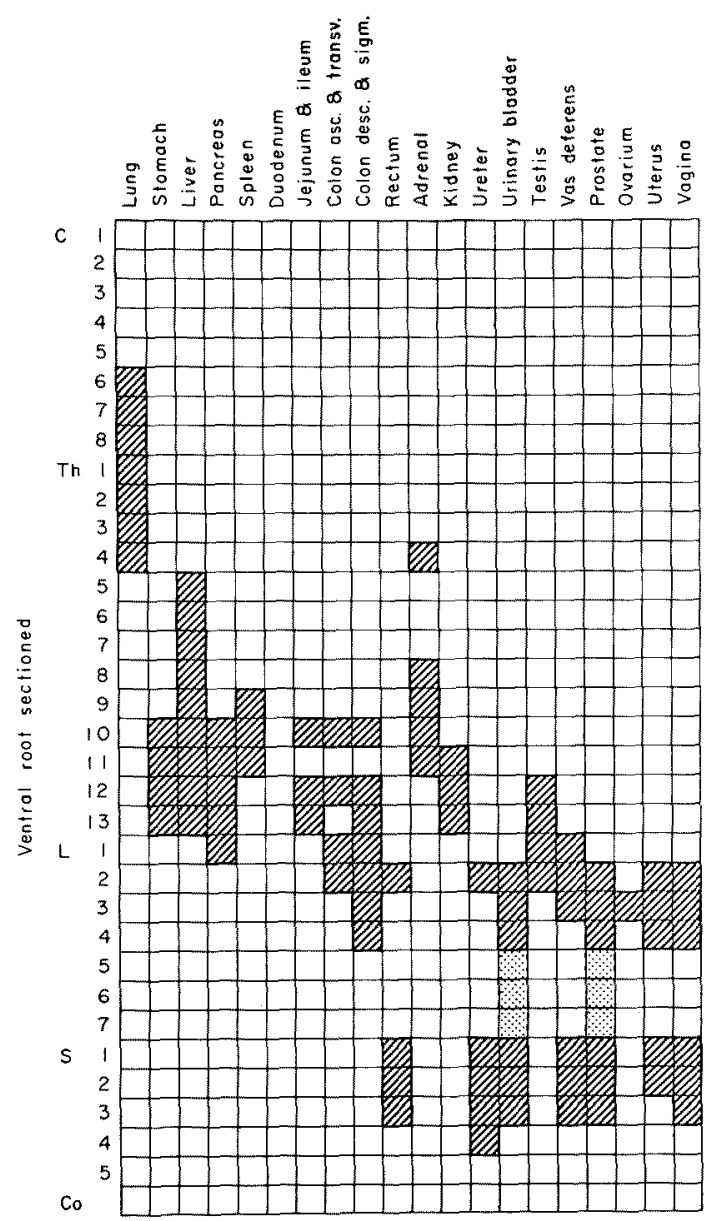

Fig. 6. The distribution of the degenerated nerve fibers to the visceral organ after the section of the ventral root. The hatched area shows the presence of degenerated fibers.

the nodose ganglion. The results obtained are shown in Table 1, with number of the degenerated nerve fibers in two representative cases. And it was clarified that the vagus nerve has also rather wide innervation to every visceral organ.

In practice of spinal anesthesia, it is important to know the correlation between the dermatomic level of anesthesia and the anesthetised region of the visceral organs. And we can suppose a little difference between them from the results mentioned above, especially in the autonomic system. However, the entity could not be clarified enough in this experiment because of the nature of experimental procedure, and it awaits further studies.

\section{References}

1) Chan Hung-I (1957) Experimental and histological studies on the thoraco-lumbar parts 


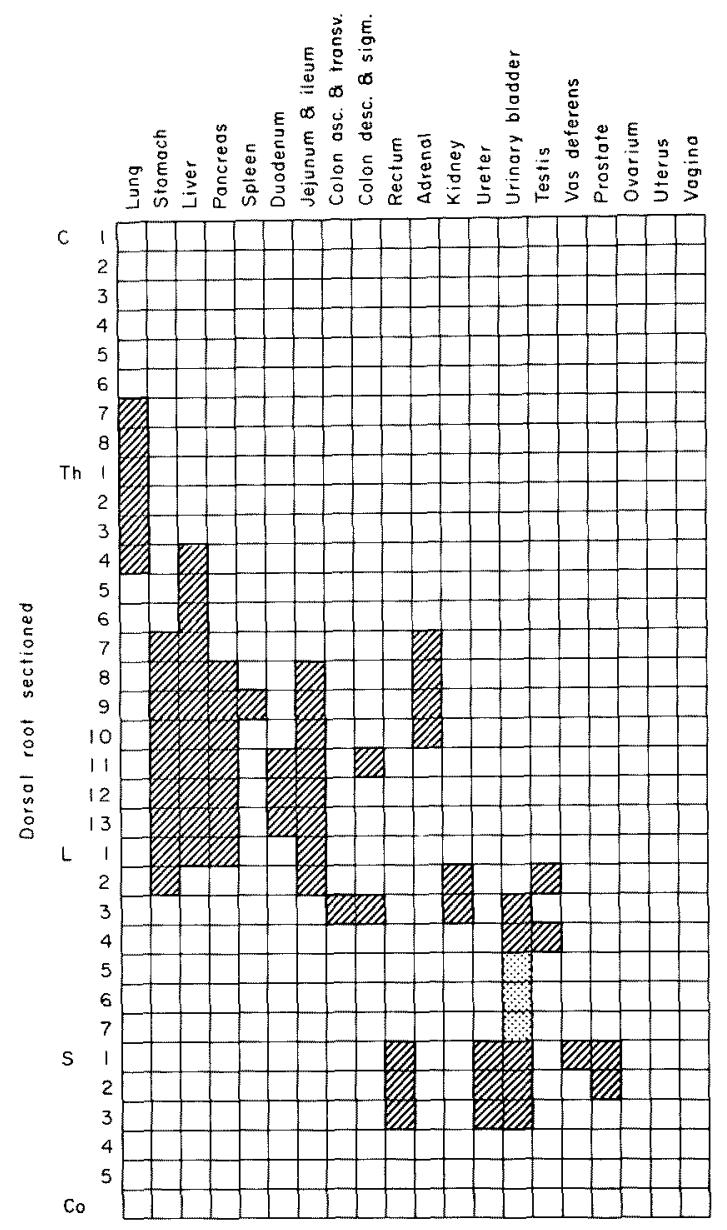

Fig. 7. The distribution of the degenerated nerve fibers to the visceral organ after the section of the dorsal root. The hatched area shows the presence of degenerated fibers.

of the sympathetic trunk. Fukushima med. J., 7, 11-31. (Japanese)

2) Fukuyama, U. (1955) On the constituents of the hypogastric nerve. Fukushima J. med. Sci., 1, 117-139.

3) Funada, A. (1960) Observation on the degeneration of the myelinated nerve fiber after the section of the ventral root of the lower thoracic spinal nerve. Fukushima med. J., 10, 261-285. (Japanese)

4) Hamabe, M. (1932) On the relationship between the sensation and the diameter of various myelinated nerve fibers. Tokyo med. J., 46, 673-694. (Japanese)

5) Hara, M. (1957) Experimental and histological studies on the sensation of the stomach. Fukushima med. J., 7, 531-551. (Japanese).

6) Haruyama, C. (1958) Experimental and histological studies on the splanchnic nerves. Fukushima med. J., 8, 683-717. (Japanese)

7) Honda, T. (1960) Experimental neurohistological studies on the innervation of the liver. Fukushima med. J., 10, 181-201. (Japanese)

8) Lizuka, T. (1959) A histological study of the innervation in the lung. Fukushma med., J., 9, 525-554. (Japanese) 


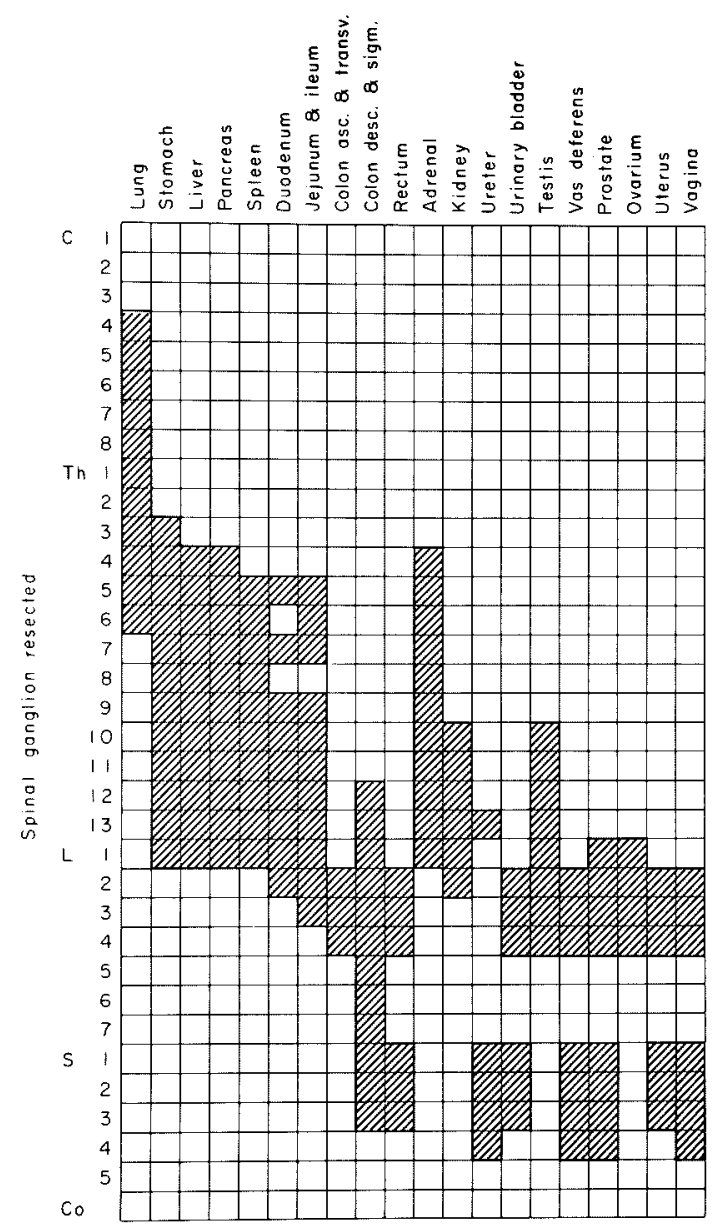

Fig. 8. The distribution of the degenerated small-sized nerve fibers to the visceral organ after the spinal ganglionectomy. The hatched area shows the presence of degenerated fibers.

9) Imabayashi, K. (1971) Experimental study on the neurogenic bladder. XVII. A new concept on the innervation of the lower lumbar spinal cord to the canine urinary bladder. Jap. J. Urol., 62, 541-559. (Japanese)

10) Inomata, S. (1957) Clinical and histological study on the sensation in the gall bladder. Fukushima med. J. 7, 69-87. (Japanese)

11) Inomata, Y. (1960) Experimental and histological studies on the sympathetic trunk. Report 5. Degeneration of myelinated fibers in the sympathetic trunk by means of the section of the dorsal root of the lumbar and sacral nerve. Fukushima med. $J ., 10,213-221$. (Japanese)

12) Irisawa, S. (1959) Experimental and histological studies on the sympathetic trunk. VI. Degeneration of the myelinated nerve fibers in the sympathetic trunk following resection of the spinal ganglion of the lumbar and sacral nerves. Fukushima med. J., 9, 501-517. (Japanese)

13) Kano, R. (1958) Experimental histological studies on the splanchnic nerves. II. On the degeneration of myelinated nerve fibers in case of the section of dorsal root of 


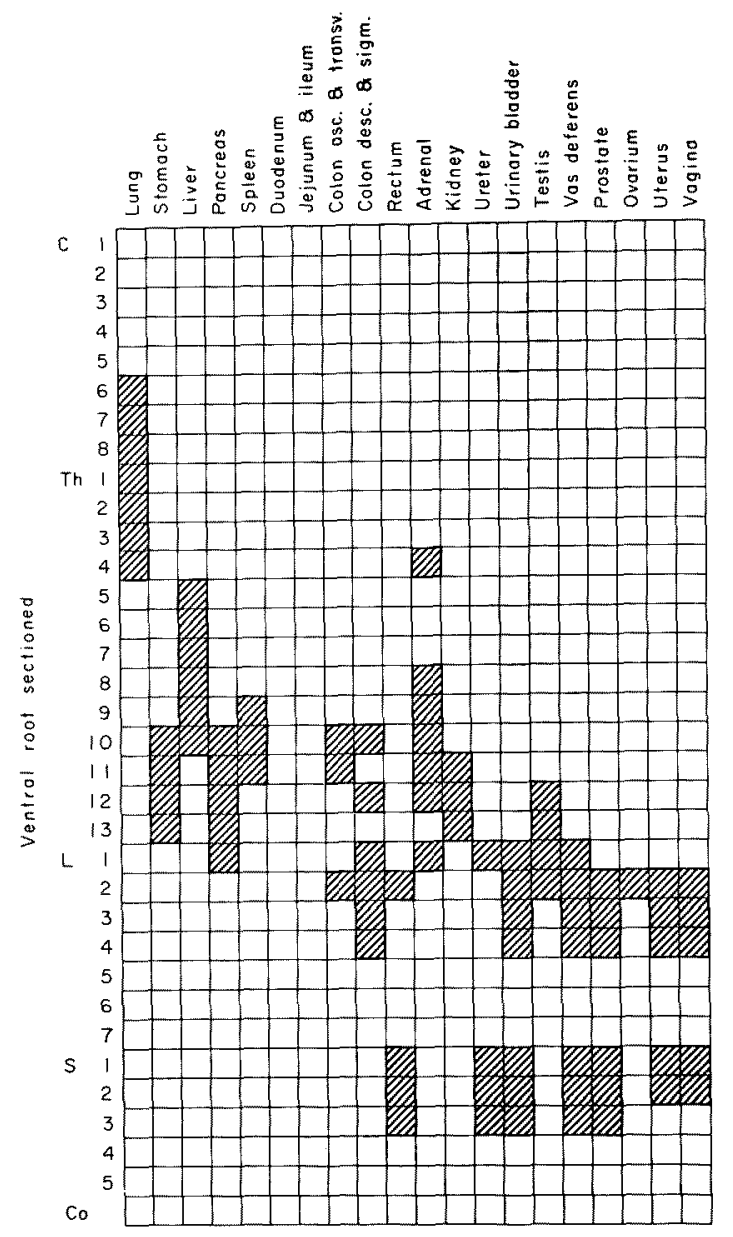

Fig. 9. The distribution of the degenerated small-sized nerve fibers to the visceral organ after the section of the ventral root. The hatched area shows the presence of degenerated fibers.

thoracic and lumbar nerves. Fukushima med. J., 8, 627-652. (Japanese)

14) Kashimura, Z. (1959) Experimental and histological studies on the thoracomumbar part of the sympathetic trunk. 2. Degeneration of myelinated fibers in the sympathetic trunk by means of the section of the dorsal root of the thoracic nerve. Fukushima med. J. 9, 147-164. (Japanese)

15) Kato, W. (1960) Degeneration of myelinated fibers by means of the section of the dorsal root of the lower thoracic nerve. Fukushima med. $J ., 10,627-649$. (Japanese)

16) Kikuchi, K. (1955) An experimental histological study on the splanchnic nerves. Fukushima med. J., 5, 353-376. (Japanese)

17) Kimura, S. (1960) Experimental and neurohistological studies on the innervation of pelvic viscera from N. hypogastricus as well as N. pelvicus. Fukushima med. J., 10, 367-402. (Japanese)

18) Koseki, S. (1958) A histological study on the innervation of the pancreas. Fukushima med. J., 8, 289-312. (Japanese)

19) Kure, K.\& Okinaka, S. (1949) Autonomic Nervous System, 5th ed., Kanehara, Tokyo, 


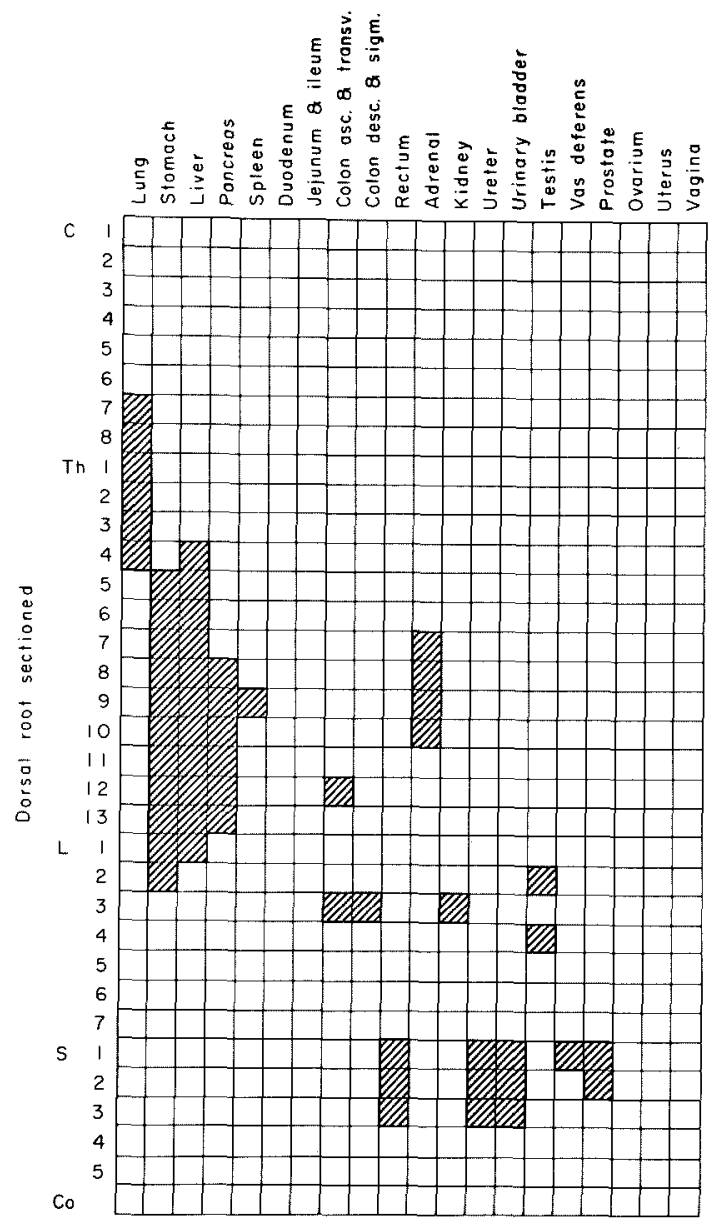

Fig. 10. The distribution of the degenerated small-sized nerve fibers to the visceral organ after the section of the dorsal root. The hatched area shows the presence of degenerated fibers.

\section{p. 54. (Japanese)}

20) Maeda, S. (1959) Experimental and histological studies on the sympathetic trunk. III. Degeneration of the myelinated nerve fibers in the thoraco-lumbar sympathetic trunk following resection of the ventral root of the thoracic nerves. Fukushima med. $J .$, 9, 555-569. (Japanese)

21) Mashiko, T. (1959) Studies on nerves innervating the adrenal glands. Fukushima med. J., 9, 621-637. (Japanese)

22) Muto, K. (1960) Histological studies on the abdominal organs of the vagus. Fukushima med. $J ., 10,307-334$. (Japanese)

23) Okane, R. (1960) Experimental and histological studies of the innervation of the ureter. Fukushima med. J., 10, 675-690. (Japanese)

24) Okuno, T. (1958) Experimental and neurohistological studies on the innervation of the male internal sexual organs. Fukushima med. J., 8, 229-262. (Japanese)

25) Okuno, T., Funada, S. \& Watanabe, S. (1958) Clinical and nurohistological studies on the innervation of the female internal sexual organs. Fukushima med. J., 8, 1-17. 
(Japanese)

26) Ono, M. (1960) Experimental and histological studies on the innervation of abdominal organs of the splanchnic nerve. Fukushima med.J., 10, 657-674. (Japanese)

27) Osaki, T. (1956) On the small-sized myelinated nerve fibers in the major and minor splanchnic nerves. Nisshin-Igaku, 43, 24-28. (Japanese)

28) Saito, T. (1958) Histological study on the sacral domination of the urinary bladder. Fukushima med. J., 8, 137-160. (Japanese)

29) Sakata, A. (1961) Experimental and histological studies of the innervation of the rectum. Fukushima med. J., 11, 171-195. (Japanese)

30) Sakauchi, Z. (1960) Observation of the degeneration of myelinated fibers after the section of the dorsal root ganglion of the lower thoracic nerve. Fukushima med. $J ., 10$, 63-89. (Japanese)

31) Sato, T. (1957) Histological studies on the lumbar innervation of the urinary bladder. Fukushima med. J., 7, 367-392. (Japanese)

32) Sato, T., Suzuki, K., Chan, H.I., Kikuchi, K., Hara, M., Koseki, S., Maeda, S., Iirsawa, S., Kano, R., Sato, W., Haruyama, C., Kashimura, Z., Inomata, Y., Funada, A. \& Sakauchi, Z. (1957) Experimental and histological studies on the pathway of the visceral sensation. Jap. J. Surg., 58, 753-754. (Japanese)

33) Shishito, S. \& Suzuki, K. (1961) On the centrifugal nerve fibers in the dorsal root of the spinal nerves innervating thoraco-abdominal and pelvic organs. Tohoku med. $J$., 63, 66-92. (Japanese)

34) Suzuki, M. (1958) Clinical and histological studies on sensation from the small intestine. Fukushima med. J., 8, 535-562. (Japanese)

35) Tokoyo, K. (1959) Clinical, experimental and histological studies on the sensation of the kidney. Fukushima med. J., 9, 129-146. (Japanese)

36) Watanabe, S. (1959) Experimental and histological studies of the innervation of the spleen. Fukushima med. J., 9, 660-685. (Japanese)

37) Yambe, J. (1960) Experimental and histological studies on the lumbo-sacral part of the sympathetic trunk. 7. Degeneration of myelinated fibers in the sympathetic trunk by means of the section of the ventral root of the lumbar and sacral nerve. Fukushima med. J., 10, 583-593. (Japanese)

38) Yoshinari, M. (1959) Experimental and histological studies of the innervation of the colon. Fukushima med. J., 9, 429-459. (Japanese) 